\title{
Wrinkled Polydimethylsiloxane for Enhanced Light Trapping and Anti-Reflection in Flexible Corrugated Silicon Solar Cells
}

\author{
Nazek El-Atab ${ }^{1}$, Wedyan Babatain ${ }^{1}$ and Muhammad M. Hussain ${ }^{1,2}$ \\ ${ }^{1} \mathrm{MMH}$ Labs, Electrical and Computer Engineering, King Abdullah University of Science and Technology \\ (KAUST), Thuwal 23955-6900, Saudi Arabia. \\ ${ }^{2}$ EECS, University of California, Berkeley, California 94720, USA
}

\begin{abstract}
Light trapping and anti-reflection schemes serve a key role in reducing light reflection and enhancing absorption within solar cells. Here, we demonstrate the use of polydimethylsiloxane (PDMS) for enhancing light confinement in flexible corrugated c-silicon solar cells. More specifically, corrugated silicon solar cells show a unique textured architecture where islands of silicon are interconnected via interdigitated back contacts. Wrinkled PDMS on the silicon islands in addition to flat PDMS anti-reflective coating within the grooves are shown to improve the power output in flexible corrugated solar cells by almost 9\%. Wrinkled PDMS is created during the $\mathrm{CO}_{2}$ laser patterning step applied on a bilayer system with a mismatch in the coefficient of thermal expansion, thus causing linear buckling. It should be noted that, in this case, PDMS serves as both a coating for encapsulating and protecting the cells from the external environmental factors such as water, mechanical shock, etc., in addition to a material for reducing light reflection.
\end{abstract}

Keywords—light trapping, silicon, flexible, wrinkles, PDMS, corrugation

\section{INTRODUCTION}

In order to improve the efficiency of solar cells, several schemes have been developed for capturing the sunlight hitting the surface of the cell instead of letting it be reflected away. As a result, photons get confined leading to a higher probability of their absorption and their conversion into electrons [1-2]. This can be generally achieved by using an anti-reflective coating with a specific index of refraction and thickness such that destructive interference is achieved between the waves reflected from the surfaces of the semiconductor and the anti-reflective coating [3], or by texturing the surface of the cell such that light bounces back and forth within the cell thus enabling multiple passes within the cell instead of being reflected [4].

Nevertheless, commercial solar cells are generally never in direct contact with the air. A polymeric material is commonly used to protest the cells from various environmental factors in addition to enhancing their mechanical robustness. The presence of the polymeric material at the surface of the cell modifies its light scattering properties [5]. While glass is mainly used for rigid modules, flexible solar cells require a flexible polymeric encapsulant. In this work, flexible c-silicon solar cells with interdigitated back contacts and which are developed using a corrugation technique are studied with a polydimethylsiloxane (PDMS) anti-reflective and light-trapping coating. The effect of the ripples and the coating within the grooves on the reflectance from the solar cell and its electrical characteristics are examined.

\section{EXPERIMENTAL METHODS}

In order to study the effect of the PDMS light trapping and anti-reflective coating on flexible solar cells, our previously demonstrated corrugation technique is used to convert rigid cells into their flexible version [6-10]. Commercial grade crystalline silicon solar cells with interdigitated back contacts are initially coated with a $200 \mu \mathrm{m}$ thick PDMS layer which is next patterned using a $\mathrm{CO}_{2}$ laser. Next, the exposed silicon areas between the PDMS patterns are completely removed in a deep reactive ion etching system (DRIE). Finally, the resulting flexible solar cells are coated again with another layer of PDMS encapsulating layer as shown in Fig. 1. In order to study the effect of the $\mathrm{CO}_{2}$ laser on the PDMS, SEM images in addition to reflectance measurements are conducted on the surface of the PDMS before and after the process.

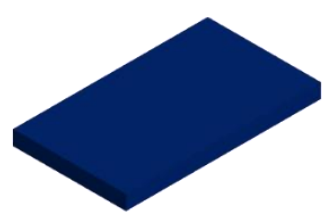

1. c-Si solar cell with IBC

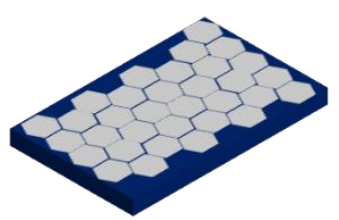

3. Patterning of PDMS and ripples generation using $\mathrm{CO}_{2}$ laser

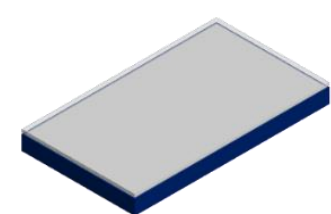

2. Coating of PDMS

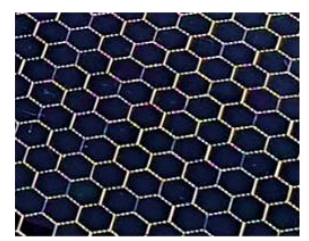

4. DRIE etching and PDMS encapsulation
Fig. 1. Illustration of the corrugation and encapsulation process applied on rigid silicon solar cells with interdigitated back contacts. 


\section{RESULTS AND DISCUSSIONS}

The SEM images show a rough surface of the reference PDMS while a dense array of ripples is obtained on the surface after applying the $\mathrm{CO}_{2}$ laser patterning process as depicted in Fig. 2. In fact, during the patterning process, the system composed of the silicon cell and the PDMS surface heat up and expand. Linear buckling leading to the formation of ripples takes place to minimize the total energy of the system when the compressive stress exceeds a critical threshold value [11]. To study the effect of the ripples of the reflection properties of the PDMS surface, two samples are prepared where flat PDMS and rippled PDMS are added on a PMMA sheet with a back reflector. Diffuse reflection is measured using a PerkinElmer Lambda 950 UV-Vis-NIR system (150 mm integrated sphere). The reflectance measurements show that the wrinkles contribute to reducing the reflectance by around $4 \%$ compared to a reference rough PDMS surface as shown in Fig. 3. Therefore,

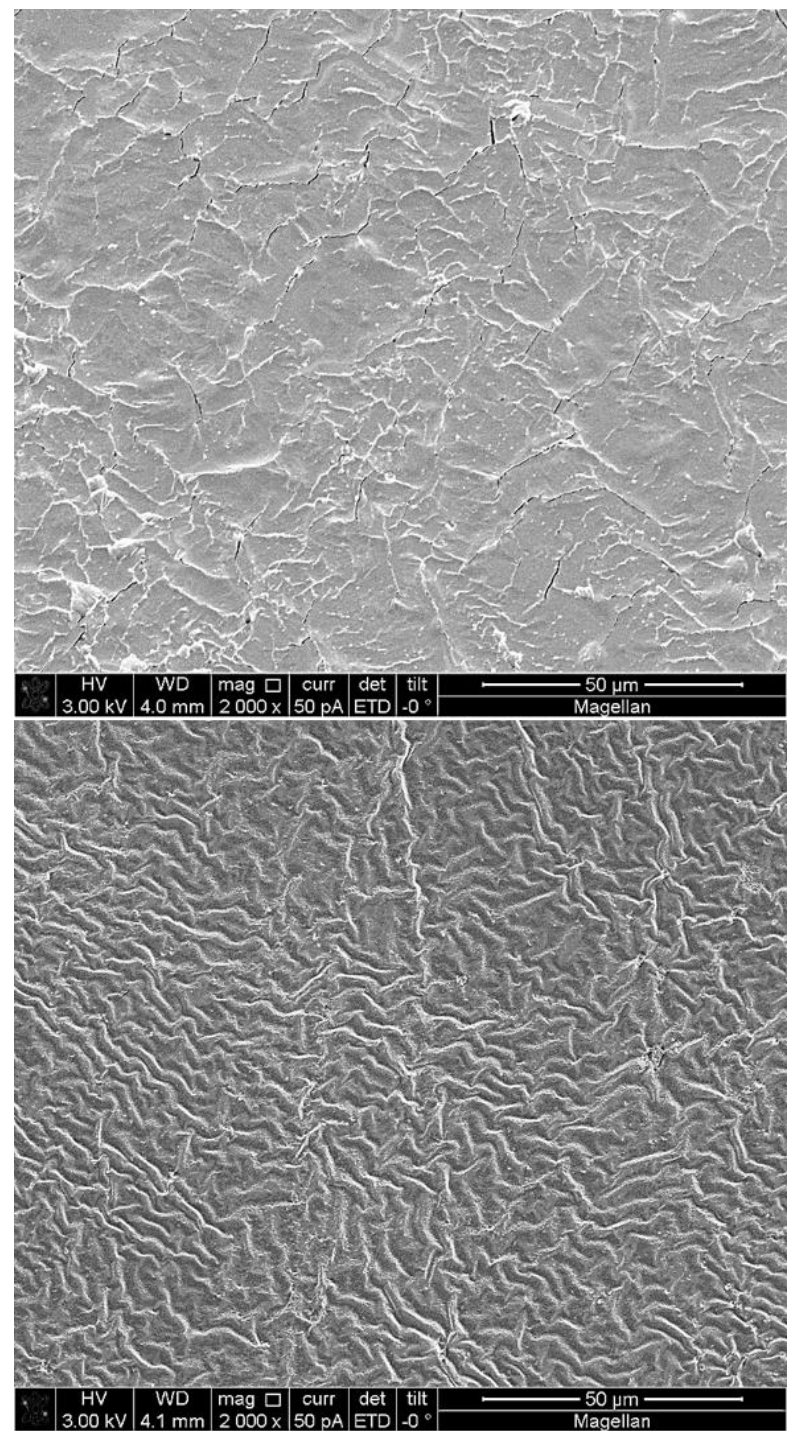

Fig. 2. SEM images of the PDMS surface before (top) and after (bottom) patterning it using the $\mathrm{CO}_{2}$ laser. Ripples are created to minimize the overall energy of the system with different coefficient of thermal expansion.

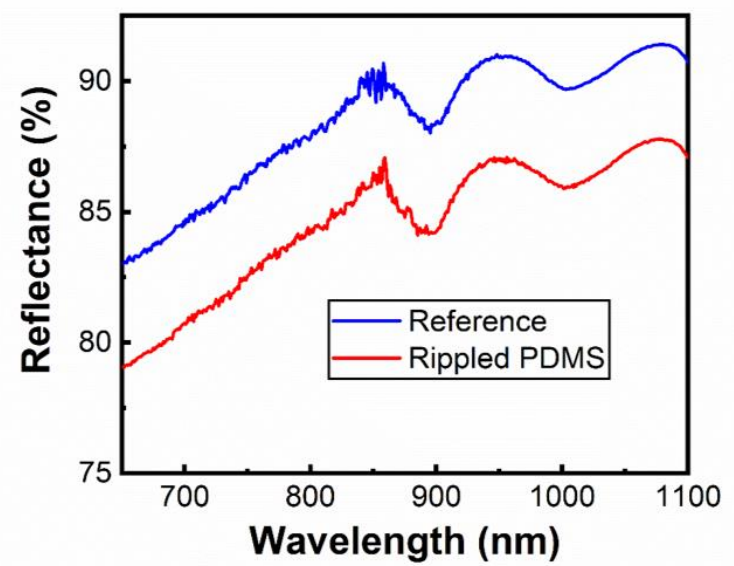

Fig. 3. Reflectance spectrum of flat reference PDMS vs. the ripples PDMS when applied on a 2-mm PMMA sheet with a copper back reflector.

the patterning of the PDMS applied on the rigid solar cells using the $\mathrm{CO}_{2}$ laser leads to rippled PDMS surfaces on the silicon islands and thus enhance the light trapping properties of the cells.

Moreover, unlike commonly available flexible solar cells which generally have a flat surface, the corrugated cells are unique in that islands of silicon are interconnected via interdigitated back contacts with $135 \mu \mathrm{m}$ wide and $375 \mu \mathrm{m}$ deep grooves in between the islands [6-8]. As a result, the use of a conformal anti-reflective coating on the cell can enhance the light trapping within the grooves due to the already textured surface of the cell. PDMS is selected as the coating layer to serve as both an encapsulation material which protects the cell from the various outer environmental factors and improves the mechanical resilience of the cells, in addition to reducing light reflection. As a matter of fact, reflection can be reduced if the refractive index of the anti-reflective coating is the geometric mean of the refractive indices of the surrounding materials. In the case of the silicon solar cells with $\mathrm{IBC}, \mathrm{Si}_{3} \mathrm{~N}_{4}$ and air are the surrounding materials which makes PDMS the perfect antireflective material [2-4].

In order to explore the effect of the PDMS encapsulation layer as an anti-reflective coating on corrugated solar cells, the reflectance spectrum is measured on the encapsulated and unencapsulated versions of solar cells with two different corrugation patterns: diamond and honeycomb. The encapsulation layer in this case covers both the silicon areas in addition to the grooves with interdigitated back contacts. As depicted in Fig. 4, the encapsulation is shown to reduce the reflectance by up to $3 \%$ in the case of the honeycomb patterned cell while $2 \%$ with the diamond patterned cell. This can be explained by the increased loss of active silicon area in the honeycomb patterned cell compared with the diamond patterned cell $(12 \%$ vs. $6 \%)$ and thus the increased exposed area of the back contacts reflecting light.

The effect of the PDMS as a light trapping and anti-reflective coating in corrugated silicon solar cells is studied using a solar simulator in air under $100 \mathrm{~mW} / \mathrm{cm}^{2}$. The PDMS ripples and antireflective coating are found to enhance the short circuit current 

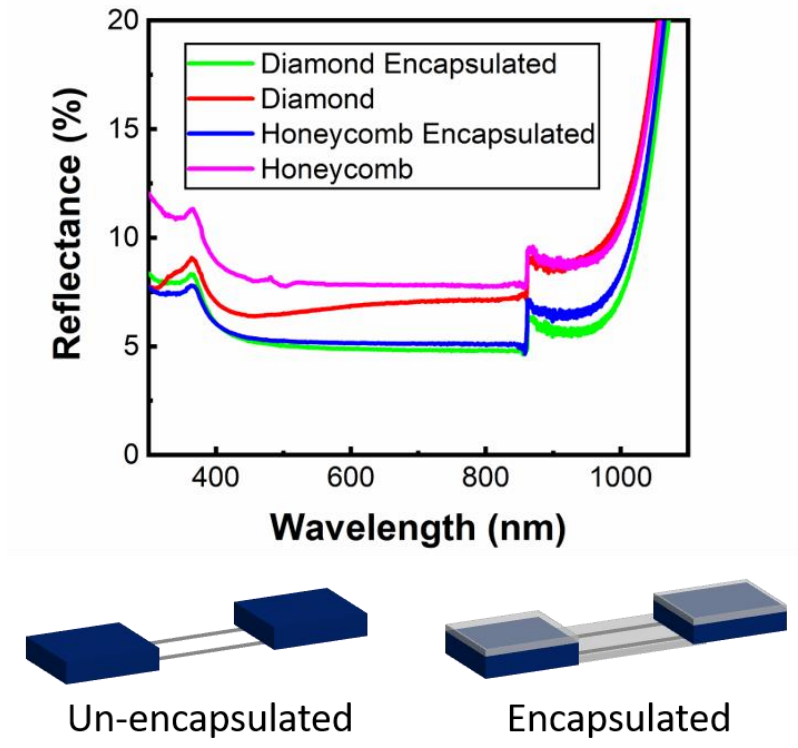

Fig. 4. Top, Reflectance spectrum of the un-encapsulated vs. encapsulated corrugated solar cells with different patterns (diamond and honeycomb). Bottom, Schematic illustration of the un-encapsulated vs. encapsulated corrugated solar cells.

and the power output by almost $9 \%(0.603 \mathrm{~W})$ compared to a reference cell $(0.554 \mathrm{~W})$ as shown in Table I.

\section{CONCLUSION}

In conclusion, the role of PDMS in trapping light and reducing the light reflection at the surface of corrugated flexible solar cells is demonstrated. Wrinkles in PDMS are created during the patterning process using a $\mathrm{CO}_{2}$ laser inducing linear buckling as a result of the generated large compressive stress in a system with a mismatch in the coefficient thermal expansion. This leads to the formation of ripples on the silicon islands. In addition, the encapsulation of the corrugated cells with a PDMS layer serves in reducing light reflection due to refractive index in addition to contributing to the protection of the cells from water, snow, and other environmental factors and improving their mechanical robustness. Finally, the results show that an increase in the power output of the corrugated flexible solar cells

TABLE I

ELECTRICAL CHARACTERISTICS OF THE REFERENCE VS. PDMS ENCAPSULATED

\begin{tabular}{|c|c|c|c|c|}
\hline & $\mathbf{V}_{\text {oc }}(\mathbf{V})$ & $\mathbf{I}_{\text {sc }}(\mathbf{A})$ & $\mathbf{F F}(\%)$ & $\mathbf{P}_{\max }(\mathbf{W})$ \\
\hline Reference Cell & 0.638 & 1.15 & 75.5 & 0.554 \\
\hline Encapsulated Cell & 0.64 & 1.24 & 75.9 & 0.603 \\
\hline
\end{tabular}

by almost $9 \%$ can be achieved with the addition of the PDMS ripples and PDMS encapsulation layer.

\section{ACKNOWLEDGMENT}

This publication is based upon work supported by the King Abdullah University of Science and Technology (KAUST) Office of Sponsored Research (OSR) under Award No. Sensor Innovation Initiative OSR-2015-Sensors-2707 and KAUSTKFUPM Special Initiative OSR-2016-KKI-2880.

\section{REFERENCES}

[1] J. Zhu, C. Hsu, Z. Yu, S. Fan and Y. Cui, "Nanodome Solar Cells with Efficient Light Management and Self-Cleaning", Nano Letters, vol. 10, no. 6, pp. 1979-1984, 2010. Available: 10.1021/n19034237.

[2] E. Garnett and P. Yang, "Light Trapping in Silicon Nanowire Solar Cells", Nano Letters, vol. 10, no. 3, pp. 1082-1087, 2010. Available: 10.1021/nl100161z.

[3] S. Mallick, M. Agrawal and P. Peumans, "Optimal light trapping in ultrathin photonic crystal crystalline silicon solar cells", Optics Express, vol. 18, no. 6, p. 5691, 2010. Available: 10.1364/oe.18.005691.

[4] C. Lin, M. Tsai, W. Wei, K. Lai and J. He, "Packaging Glass with a Hierarchically Nanostructured Surface: A Universal Method to Achieve Self-Cleaning Omnidirectional Solar Cells", ACS Nano, vol. 10, no. 1, pp. 549-555, 2015. Available: 10.1021/acsnano.5b05564.

[5] A. Amalathas and M. Alkaisi, "Upright nanopyramid structured cover glass with light harvesting and self-cleaning effects for solar cell applications", Journal of Physics D: Applied Physics, vol. 49, no. 46, p. 465601, 2016. Available: 10.1088/0022-3727/49/46/465601.

[6] N. El-Atab, W. Babatain, R. Bahabry, R. Alshanbari, R. Shamsuddin and M. Hussain, "Ultraflexible Corrugated Monocrystalline Silicon Solar Cells with High Efficiency (19\%), Improved Thermal Performance, and Reliability Using Low-Cost Laser Patterning", ACS Applied Materials \& Interfaces, 2019. Available: 10.1021/acsami.9b15175.

[7] N. El-Atab, N. Qaiser, R. Bahabry and M. Hussain, "Corrugation Enabled Asymmetrically Ultrastretchable (95\%) Monocrystalline Silicon Solar Cells with High Efficiency (19\%)", Advanced Energy Materials, vol. 9, no. 45, p. 1902883, 2019. Available: 10.1002/aenm.201902883.

[8] N. El-Atab, N. Qaiser, W. Babatain, R. Bahabry, R. Shamsuddin and M. Hussain, "Nature-inspired spherical silicon solar cell for threedimensional light harvesting, improved dust and thermal management", MRS Communications, vol. 10, no. 3, pp. 391-397, 2020. Available: $10.1557 / \mathrm{mrc} .2020 .44$.

[9] N. El-Atab, S. Khan and M. Hussain, "Flexible High-Efficiency Corrugated Monocrystalline Silicon Solar Cells for Application in Small Unmanned Aerial Vehicles for Payload Transportation", Energy Technology, vol. 8, no. 11, p. 2000670, 2020. Available: 10.1002/ente.202000670.

[10] N. El-Atab and M. Hussain, "Flexible and stretchable inorganic solar cells: Progress, challenges, and opportunities", MRS Energy \& Sustainability, vol. 7, 2020. Available: 10.1557/mre.2020.22.

[11] F. Li, H. Hou, J. Yin and X. Jiang, "Near-infrared light-responsive dynamic wrinkle patterns", Science Advances, vol. 4, no. 4, p. eaar5762, 2018. Available: $10.1126 /$ sciadv.aar5762. 